\title{
Selected acute phase CSF factors in ischemic stroke: findings and prognostic value
}

\author{
Maia Beridze ${ }^{1 *}$, Tamar Sanikidze ${ }^{2}$, Roman Shakarishvili1 ${ }^{1}$, Nino Intskirveli ${ }^{2}$ and Natan M Bornstein ${ }^{3}$
}

\begin{abstract}
Background: Study aimed at investigation of pathogenic role and prognostic value of several selected cerebrospinal fluid acute phase factors that can reflect the severity of ischemic brain damage.

Methods: Ninety five acute ischemic stroke patients were investigated. Ischemic region visualized at the twenty fourth hour by conventional Magnetic Resonance Imaging. Stroke severity evaluated by National Institute Health Stroke Scale. One month outcome of disease was assessed by Barthel Index. Cerebrospinal fluid was taken at the sixth hour of stroke onset. CSF pro- and anti-inflammatory cytokines were studied by Enzyme Linked Immunosorbent Assay. Nitric Oxide and Lipoperoxide radical were measured by Electron Paramagnetic Resonance. CSF Nitrate levels were detected using the Griess reagent. Statistics performed by SPSS-11.0.

Results: At the sixth hour of stroke onset, cerebrospinal fluid cytokine levels were elevated in patients against controls. Severe stroke patients had increased interleukin- 6 content compared to less severe strokes $(P<0.05)$. Cerebrospinal fluid Electron Paramagnetic Resonance signal of nitric oxide was increased in patients against controls. Severe stroke group had an elevated Electron Paramagnetic Resonance signal of lipoperoxiradical compared to less severe stroke. Cerebrospinal fluid nitrate levels in less severe stroke patients were higher than those for severe stroke and control. Positive correlation was established between the initial interleukin- 6 content and ischemic lesion size as well as with National Institute Health Stroke Scale score on the seventh day. Initial interleukin- 6 and nitrate levels in cerebrospinal fluid found to be significant for functional outcome of stroke at one month.
\end{abstract}

Conclusion: According to present study the cerebrospinal fluid contents of interleukin- 6 and nitrates seem to be the most reliable prognostic factors in acute phase of ischemic stroke.

Keywords: brain ischemia, inflammation, oxidative stress

\section{Background}

Modern concepts of acute cerebral ischemia highlight the role of neurovascular units and emphasize the importance of integrative tissue responses that result from dynamic interactions of endothelial cells, vascular sooth muscles, matrix elements, astroglia, microglia and neurons. By means of inflammatory stimuli and excitoxicity, such interactions create many sources of free toxic radicals and reactive oxygen spices [1].

In physiological conditions, endogenous protective mechanisms stabilize the levels of free oxygen radicals

\footnotetext{
* Correspondence: postdoc2002@yahoo.com

'Department of Neuromedicine, Tbilisi State Medical University, Tbilisi, Georgia

Full list of author information is available at the end of the article
}

and reduce the oxidative/nitrosative stress reaction. In conditions of severe ischemia, rapid failure of the antioxidation protective system assists in the accumulation of arachidonic acid, prostaglandins, superoxide anion, $\mathrm{NO}$ and other aggressive substrates, which lead to the destabilization of cellular membranes, further damage of the blood-brain barrier, disintegration of DNA and, ultimately, to neuronal death [2]. Current therapeutic options for acute brain ischemia are concentrated on thrombolytic treatment, but this therapy is restricted to a small proportion of patients [3]. There is a need to devise a more effective protective and repair strategy and cellular treatment. The precise neurochemical alterations that take place in human's stroke still remain to be clarified and the cerebrospinal fluid (CSF) is the

\section{Biomed Central}


closest environment reflecting the immediate immunobiochemical changes in the ischemic brain tissue. The purpose of the present research was to investigate the importance and prognostic value of several selected CSF acute phase factors that are known to reflect the severity of ischemic brain damage.

\section{Methods}

A total of 95 acute ischemic stroke patients, 54 female and 41 male, aged 45-70 years, who had been admitted to the Neurological Clinic of Tbilisi State Medical University during 2005-2009 were studied. Exclusion criteria comprised acute inflammatory and autoimmune disorders, severe somatic pathology, cancer, coma, space occupying hemispheric and cerebellar ischemic strokes. Patients and controls that used the anti-inflammatory medications for the 1-year period prior to the current research were also excluded from the study. Upon admission, a conventional CT scan was performed to exclude a brain hemorrhage. The control group consisted of 25 age-matched patients with vertebral discopathies, who showed no signs of cerebrovascular pathology. The local ethics committee approved the protocol, and informed consent was obtained from all participants or their surrogates

The etiology of stroke was classified according to TOAST criteria [4]. Medical records were retrospectively reviewed for selected non-modifiable and modifiable risk factors of stroke, including age, sex, inheritance, history of a transient ischemic attack (TIA) or a previous stroke, hypertension, atherosclerosis, atrial fibrillation, diabetes mellitus, smoking, alcohol abuse, acute infections 1-2 months before stroke, and psychological stress. The latter was ascertained by self-report in refuges from problematic regions of Georgia. Psychological stress was of interest to determine whether it can influence the inflammatory markers or functional outcome of the disease $[5,6]$.

Upon admission, body temperature and blood pressure were recorded. Next, chemistry, basic hematology, chest $\mathrm{X}$-rays, and electrocardiography were performed. Selected patients did not display marked hyperthermia or infective complications. Patients were managed according to evidence-based stroke guidelines. Thrombolytics, hemodilution, corticoids, and Nimodipine were not applied. Patients were strictly controlled and administered for hypo- and hyperglycemia and hypertension. Antiplatelet drugs were used in atherothrombotic and lacunar infarcts and anticoagulants -in suspected cardioembolic infarcts when the initial CT or magnetic resonance imaging (MRI) scans did not show a large cerebral lesion.

The ischemic region was visualized at the twentyfourth hour from stroke onset by conventional magnetic resonance imaging (MRI; magnet operating 1,5 Tesla, Vision, Siemens) that provided axial T1, T2 images with a slice thickness of $5 \mathrm{~mm}$. Radiologists who were blinded to study protocol defined the whole lesion volume by multiplying the area of focal hyperintensity by interslice gap. Stroke severity at admission and on the seventh day was evaluated by the National Institute Health Stroke Scale (NIHSS). Patients were divided in two groups: Group 1 included the patients with severe stroke (NIHSS $\geq 15$ ) and Group 2 included the patients with mild and moderate stroke (NIHSS <15) [7]. Functional outcome was evaluated by Barthel Index at 1 month of stroke onset (BI) $[8,9]$.

\section{Immunological Assay}

For special laboratory investigations, $10 \mathrm{ml}$ of CSF was taken from patients and controls at the sixth hour from stroke onset. Eight-ml CSF samples were frozen at $-20^{\circ} \mathrm{C}$ for further assays, and the other $2 \mathrm{ml}$ of CSF were frozen in liquid nitrogen for electron paramagnet resonance (EPR) study. CSF $(5 \mathrm{ml})$ levels of the proinflammatory cytokines: interleukin-1 $\beta(\mathrm{IL}-1 \beta)$, interleukin-6(IL-6), tumor necrosis factor- $\alpha$ (TNF- $\alpha)$ and the anti-inflammatory cytokine interleukin-10 (IL-10) were detected by enzyme-linked immunosorbent assay (ELISA), by application of ELISA- RIDER. The relationship between optical density and cytokine concentrations was defined using the standard curve according to kit instructions (Bender Med systems Diagnostics, Vienna, Austria).

\section{Electron Paramagnetic Resonance (EPR) Study}

Nitric oxide (NO) and lipoperoxide radical (LOO-) were measured by EPR spin labeling (radiospectrophotometer ESR-231 (X-band), with a modulation frequency of $50 \mathrm{KHZ}$ and a TM-110 cavity). Diethyldithiocarbamic acid (DETC) (Sigma) was used as an NO trap. CSF samples were incubated with Fe2+(DETC)2 stock solution. The $0.8 \mathrm{mM} \mathrm{Fe} 2+$ (DETC) colloid solution formed was yellow-brown in color and was used immediately after preparation. EPR specters of $\mathrm{NO}-\mathrm{Fe} 2+$ (DETC) 2 complexes were defined at the temperature of liquid nitrogen on a microwave power of $20 \mathrm{mVt}$. The amount of detected NO was determined from the calibration curve for integral intensity of the EPR signal of NOFe2+ (DETC)2, prepared at various concentrations (1-20 $\mu \mathrm{M})$ of the NO-donor MAHMANONOate [10]. LOO- trap, $\alpha$-phenil-tert-butilitron (PBN) (SIGMA) was used at a dosage of $50 \mathrm{ml} / 0.5 \mathrm{ml} \mathrm{CSF}$. EPR specters of LOOwere defined at room temperature on a microwave power of $20 \mathrm{mVt}$. EPR signals of LOO- were measured in arbitrary units (a.u.) (signal intensity in millimeters represented milliliters of CSF matter) [11]. 


\section{Biochemical Assay}

For nitrate (NO2) detection, $3 \mathrm{ml}$ CSF samples were processed by $20 \%$ Griess reagent. We used a CF- 46 LOMO spectrophotometer for colorimetric detection. Optical density was detected on a $540 \mathrm{~nm}$ wavelength. $\mathrm{NaNO} 2(5 \mu \mathrm{mol} / \mathrm{L})$ was used for drawing the calibrating curve [12].

\section{Statistics}

The obtained data were analyzed using SPSS 11.0 computer software. Normally distributed continuous variables were compared with repeated measure ANOVA, and the Kruskall-Wallis test compared abnormally distributed variables. The $\chi^{2}$-test was used to assess associations among categorical variables. The effect of acetyl salicylic acid (aspirin) and HMG-CoA reductase inhibitors (statins) was separated by partial correlation analysis. Spearman rank correlation and multiple logistic regression (forward stepwise conditional model) was used when all acute phase factors and stroke risk factors were entered into the model. Aspirin and Statins included in regression analysis as categorical covariate variables. The Hosmer and Lemeshow test was used to assess the goodness of fit of each model.

\section{Results}

The main characteristics of each clinical group are presented (Table 1). At the sixth hour from stroke onset, the CSF proinflammatory cytokine levels in both study groups were elevated compared to the control $(P<$ $0.05)$. There was no significant difference in IL- $1 \beta$ and TNF- $\alpha$ contents between the two groups, while Group 1 had significantly increased IL-6 contents compared with Group $2(P<0.05)$. The anti-inflammatory cytokine IL10 levels were not significantly elevated in the two study groups compared to the control $(P<0.07)$, although there was a trend towards an increase in Group 2 (Table 2).

The EPR signal intensity of $\mathrm{NO}$ was increased in Groups 1 and 2 compared to the Control $(P<0.05)$, but not between the study groups $(P<0.50)$ (Figure 1$)$. The EPR signal intensity of LOO- in Groups 1 and 2 was significantly increased compared to the control $(P<$ 0.05 ), and Group 1 had an elevated EPR signal intensity of LOO- compared to Group $2(P<0.05)$ (Figure 2). The NO2 levels for Group 2 were higher than those for Group 1 and the controls (Table 2). At the twentyfourth hour from stroke onset, the mean ischemic lesion size $\left(\mathrm{cm}^{3}\right)$ for Group 1 was significantly increased as compared to Group 2 (Table 1). There was a significant positive correlation between the initial IL- 6 contents and ischemic lesion size $(r=+0.34 ; P<0.05)$. The effect of Aspirin and Statins did not change the zero ordered correlations between study variables. Multivariate
Table 1 Main Characteristics of ischemic stroke patients of Group 1 and 2

\begin{tabular}{|c|c|c|}
\hline Characteristics & $\begin{array}{l}\text { Group } 1 \\
(\mathrm{n}=44)\end{array}$ & $\begin{array}{l}\text { Group } 2 \\
(\mathrm{n}=51)\end{array}$ \\
\hline Male (\%) & 65 & 60 \\
\hline Age (years) & $57.3(12.2)$ & $57.1(12.9)$ \\
\hline Inheritance (\%) ** & 37.8 & 14.7 \\
\hline History of TIA or previous stroke $(\%)^{* *}$ & 18.9 & 6.4 \\
\hline History of myocardial infarction (\%) & 4.2 & 2.1 \\
\hline $\mathrm{SBP}, \mathrm{mm} \mathrm{Hg}$ & $160.6(25.5)$ & $\begin{array}{l}165.8 \\
(30.8) \\
\end{array}$ \\
\hline $\mathrm{DBP}, \mathrm{mm} \mathrm{Hg}$ & $90.2(15.4)$ & $92.4(14.6)$ \\
\hline Body mass index, $\mathrm{kg} / \mathrm{m}^{2}$ & $27.2(5.8)$ & $28.4(2.6)$ \\
\hline Total/HDL cholesterol, ratio & $4.3(1.2)$ & $3.9(1.4)$ \\
\hline Triglycerides, mg/dL & $136(69)$ & $131(72)$ \\
\hline $\mathrm{C}$ reactive protein, $\mathrm{mg} / \mathrm{L}$ & $3.9(1.2)$ & $3.1(0.6)$ \\
\hline Atrial fibrillation (\%) * & 17.8 & 12.6 \\
\hline Serum glucose, $\mathrm{mg} / \mathrm{dL}$ & $\begin{array}{l}157.1 \\
(58.6) \\
\end{array}$ & $156.2(59.9$ \\
\hline Smoking (\%) & 32.6 & 35.7 \\
\hline Alcohol abuse (\%) & 4.2 & 5.3 \\
\hline $\begin{array}{l}\text { Acute infections 1-2 months before stroke } \\
(\%)\end{array}$ & 3.1 & 4.2 \\
\hline Psychological stress (\%)* & 17.8 & 11.5 \\
\hline Aspirin usage in current stroke $\%$ & 32.6 & 34.8 \\
\hline $\begin{array}{l}\text { HMG-CoA reductase inhibitors in current } \\
\text { stroke (\%) }\end{array}$ & 10.5 & 8.4 \\
\hline Fibrinogen, $\mathrm{mg} / \mathrm{dL}$ & $\begin{array}{c}422.1 \\
(100.2)\end{array}$ & $\begin{array}{c}410.4 \\
(106.8)\end{array}$ \\
\hline Leukocyte count, $\times 10^{9} / \mathrm{L}$ & $8.8(2.2)$ & $7.9(2.6)$ \\
\hline Temperature at admission $\left(\mathrm{C}^{\circ}\right)$ & $37.0(1.4)$ & $36.9(1.7)$ \\
\hline Ischemic lesion volume $(\mathrm{cm} 3)^{*}$ & $88.8(11.7)$ & $41.7(9.6)$ \\
\hline NIHSS score at admission ** & $20.2(4.1)$ & $8.6(4.9)$ \\
\hline NIHSS score on $7^{\text {th }}$ day ** & $18.5(3.2)$ & $7.3(3.5)$ \\
\hline \multicolumn{3}{|l|}{ Infarct topography } \\
\hline Cortical $(\%)^{* *}$ & 30.5 & 17.8 \\
\hline Subcortical (\%) ** & 16.8 & 35.7 \\
\hline \multicolumn{3}{|l|}{ Stroke etiology } \\
\hline Large-artery atherosclerosis (\%) & 12.9 & 8.4 \\
\hline Cardioembolism (\%) ${ }^{* *}$ & 28.4 & 13.6 \\
\hline Small vessel occlusion (lacunar) $(\%)^{* *}$ & 0 & 25.2 \\
\hline Other determined etiology (\%) & 3.1 & 2.1 \\
\hline Undetermined etiology (\%) & 3.1 & 4.2 \\
\hline
\end{tabular}

Numbers represent mean (SD) or percentage as appropriate. NIHSS values represent median (interquartil range).

${ }^{*} P<0.05 * * P<0.001$.

logistic regression analysis established a level of significance of IL- 6 contents toward the mean predicted probability of ischemic lesion size at the twenty-fourth hour from stroke onset, after all the acute phase factors and risk factors that we examined were entered into the 
Table 2 Comparison of selected CSF acute phase factors in clinical groups and control at the sixth hour from stroke onset

\begin{tabular}{|c|c|c|c|c|c|c|c|}
\hline & $\begin{array}{l}\mathrm{IL}-1 \beta \\
(\mathrm{pg} / \mathrm{ml})\end{array}$ & $\begin{array}{l}\mathrm{IL}-6 \\
(\mathrm{pg} / \mathrm{ml})\end{array}$ & $\begin{array}{l}\text { TNF- } \alpha \\
(\mathrm{pg} / \mathrm{ml})\end{array}$ & $\begin{array}{l}\mathrm{IL}-10 \\
(\mathrm{pg} / \mathrm{ml})\end{array}$ & $\begin{array}{l}\mathrm{NO}_{2} \\
(\mu \mathrm{mol} / \mathrm{L})\end{array}$ & $\begin{array}{l}\mathrm{NO} \\
(\mu \mathrm{mol} / \mathrm{L})\end{array}$ & $\begin{array}{l}\text { LOO } \\
\text { (a.u) }\end{array}$ \\
\hline Control & ${ }^{*} 0.95 \pm 0.02$ & $* 1.9 \pm 0.09$ & $* 14 \pm 2.3$ & $3.6 \pm 1.2$ & $*^{* 102 \pm 15.9}$ & $* 2.78 \pm 0.16$ & 0 \\
\hline Group I & $34.14 \pm 4.7$ & $* 58 \pm 4.6$ & $44 \pm 5.4$ & $5.9 \pm 1.4$ & $* 121 \pm 4.56$ & $33.8 \pm 7.1$ & ${ }^{*} 18 \pm 4.1$ \\
\hline Group II & $30.4 \pm 7.3$ & ${ }^{*} 21.8 \pm 4.4$ & $39.4 \pm 9.4$ & $7.1 \pm 1.2$ & $* 158 \pm 3.13$ & $30.18 \pm 6.8$ & $* 34 \pm 7.1$ \\
\hline
\end{tabular}

Data are expressed as means (SD).

model (Figure 3). A positive correlation was established between the initial IL- 6 CSF levels and the NIHSS scores on the seventh day of stroke $(\mathrm{r}=+0.52 ; \mathrm{P}<$ $0.05)$. There was no significant correlation between the CSF inflammatory markers and cortical or sub-cortical ischemic lesion sites. Researched factors found to be dependent on each other once all of them were included in stepwise logistic regression analysis toward the stroke functional outcome. Only the initial IL-6 and $\mathrm{NO}_{2}$ levels retained significance for functional outcome of stroke at one month (Table 3), and cardiogenic strokes showed borderline significance $(P=0.057)$. Effect and interactions of Aspirin and Statins were not significant in the given model. There was a negative correlation between the initial IL-6 levels and functional outcome (BI) of stroke at 1 month $(\mathrm{r}=-0.45: P<0.05)$.

\section{Discussion}

It is believed that the first local, glial immune response of the brain tissue to acute ischemia is the connection of CD4 T lymphocytes with astrocytes. Activated CD4 cells produce $\gamma$-interferon, which stimulates astrocytes to express HLA-II class antigens and to produce IL-1 $\beta$.

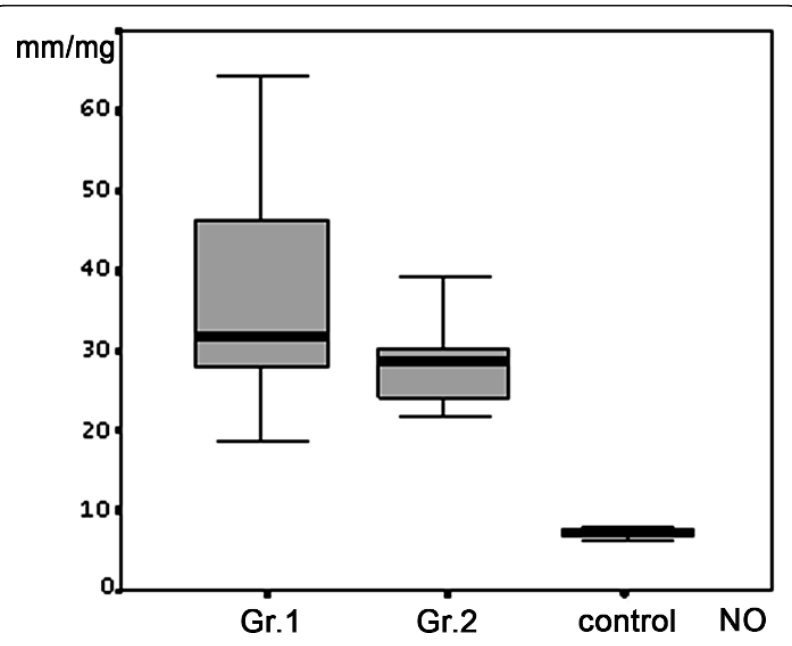

Figure 1 Comparison of EPR signal intensity of CSF free NO between the study groups and control. Box plots represent mean values (SD). $P<0.05$ between the Group1, Group 2 and control.
The latter stimulates phagocyte activity in glial tissue and induces production of IL- 6 and TNF- $\alpha$, the cytokines of initial local inflammatory reactions that trigger the subsequent development of the pro- and antiinflammatory cytokine cascade [13].

Experimental and clinical studies have demonstrated that the high CSF and blood concentrations of proinflammatory cytokines appear to reach a peak response by 24-48 hours from stroke onset [14]. The present study found elevated IL-1 $\beta$, IL- 6 and TNF- $\alpha$ level in CSF at the sixth hour from ischemic stroke onset. Previously published studies have demonstrated that elevated CSF and plasma levels of IL- $1 \beta$ correlate with larger brain infarcts and worse functional outcome $[15,16]$. The present study did not show any significant group differences in the IL- $1 \beta$ and TNF- $\alpha$ CSF levels at six hours of ischemic stroke. However, the absolute number of these cytokines was elevated in the severe stroke group, suggesting that they are of the first proinflammatory response and may trigger the subsequent proinflammatory cascade.

The significant group differences in the initial IL-6 levels and the positive correlation with the size of the ischemic region support earlier experimental and clinical studies that revealed a correlation between increased initial CSF and plasma IL-6 levels, on one hand, and larger brain lesion volume and poor outcome on the other [17]. According to a number of experimental studies, most animals retain high blood IL-6 levels during the one-year period after ischemic brain injury. IL- 6 has a mitogenic effect on astrocytes and induces reactive gliosis in later stages of brain ischemia [18]. Thus, according to the present research, IL-6 tends to reflect the severity of the stroke even at six hours post-injury and may play a key role in inflammatory damage caused by ischemia.

Previous experimental and clinical studies have shown that a high initial expression of TNF- $\alpha$ is connected with larger brain infarcts, and TNF- $\alpha$ knockout animals have larger infarcts and decreased neuronal survival [19]. Expression of TNF- $\alpha$ during the critical period of a stroke may restrict aggressive immune responses because the TNF signaling pathway involving CD95-CD95L (ligand) interactions is considered to be the controlling mechanism of $\mathrm{T}$ cell expansion during the immune response [20]. 


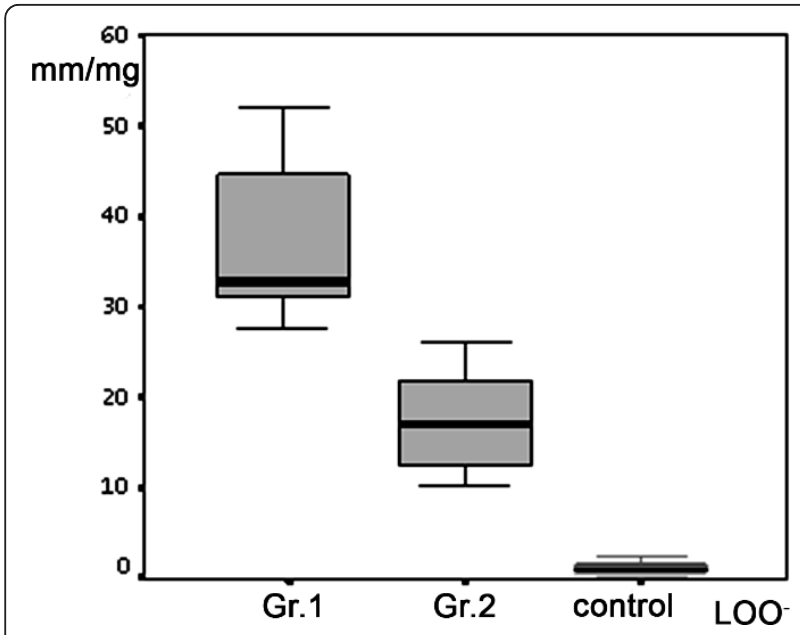

Figure 2 Comparison of EPR signal intensity of CSF free LOObetween the study groups and control. Box plots represent mean values (SD). $P<0.05$ between the study groups and control. $P<0.50$ between the Group 1 and Group 2.

As mentioned above, the CSF levels of IL-1 $\beta$ and TNF- $\alpha$ were found to be increased, but not significantly, in severe stroke patients at the time point examined in this study but might become significant at later stages of stroke. Because we could not find a significant correlation with these CSF markers and infarct size six hours after stroke onset, we hypothesize that CSF IL-6 could rapidly and specifically react in areas of ischemic damage with increased activity at later time points and retaining high meanings for a longer period. However, further studies are necessary to confirm this hypothesis.

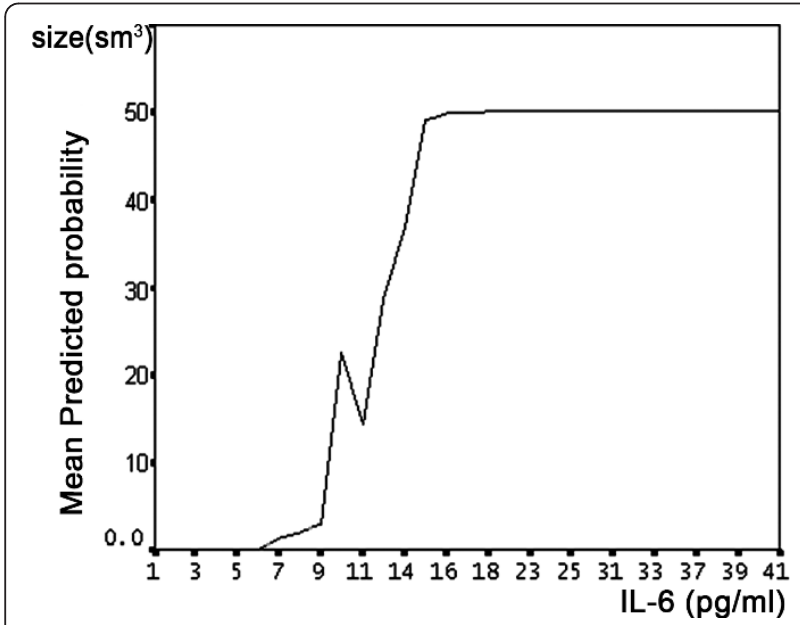

Figure 3 Relation of initial CSF IL- 6 content with ischemic lesion size $\mathbf{2 4}$ hours from stroke. After all acute phase factors and risk factors entered into the stepwise model of multivariate logistic regression only initial CSF IL-6 gained significance and positively correlated with mean predicted probability of brain ischemic lesion size $(r=+0.34 ; P<0.05)$.
Table 3 Relationship between selected acute phase factors and functional outcome of stroke at one month

\begin{tabular}{llll}
\hline $\begin{array}{l}\text { CSF Acute phase } \\
\text { factors }\end{array}$ & $\begin{array}{l}\text { Regression } \\
\text { Coefficient }\end{array}$ & $\begin{array}{l}\text { Standard } \\
\text { Error }\end{array}$ & $\boldsymbol{P}$ Value \\
\hline $\mathrm{L}-6$ & 3.08 & 0.014 & 0.031 \\
\hline $\mathrm{NO}_{2}$ & 8.06 & 2.14 & 0.001 \\
\hline
\end{tabular}

(Multivariate logistic regression analysis).

Only variables statistically significant in the final model are shown.

The anti-inflammatory cytokine IL-10 reaches its peak expression between 2-7 days after stroke onset and limits the production of proinflammatory agents through negative feedback mechanisms [21]. We could not confirm previous studies where initial IL-10 levels in blood were linked with infarct topography [22], which may be due to the relatively short time for IL-10 expression (6 hours). In keeping with previous studies, this study also connected the borderline significance of cardioembolic stroke to poor outcomes in selected patients, which might be explained by the likely association of elevated cardiac inflammatory markers in this stroke subtype [23].

In conjunction with the release of pro-inflammatory agents and glutamate toxicity, local inflammation, as described above, results in free radical pathology that directly and indirectly damages neurons by destabilizing cell membranes, disintegrating DNA, and switching the pathways of delayed neuronal death [24]. In the present study, the high EPR signal intensity of free CSF NO may be caused primarily by deregulation of the neuronal form of NO-synthase. However, NO produced by inducible and endothelial forms of NO-synthase can also pass the damaged blood-brain barrier and accumulate in CSF. The toxic effects of $\mathrm{NO}$ in the ischemic brain depend on the cellular ratio of $\mathrm{NO} / \mathrm{O}_{2}{ }^{-}$and the existence of growth factors in the surrounding tissues. The high EPR signal intensity resulting from the cell membrane lipids' degradation product, lipoperoxide radicals, LOO-, and the increased level of IL-6 in Group 1 indicates a prevalence of oxidative stress in severe stroke patients. In conditions where $\mathrm{NO}$ is more prevalent than superoxide anion $\left(\mathrm{O}_{2}^{-}\right)$, $\mathrm{NO}$ toxicity in neurons is decreased by restoration of peroxinitrite (ONOO-) to $\mathrm{NO}_{2}[25,26]$. The increased concentrations of $\mathrm{NO}_{2}$ in Group 2 patients and the relatively diminished EPR signals of LOO- indicate conditions in which NO can act as an antioxidant. The protective response of $\mathrm{NO}$ can also be obtained through nitrosonium $\left(\mathrm{NO}^{+}\right)$, which nitrosylates the thiol groups of glutamate receptors and thus diminishes glutamate toxicity [27].

The limitation of this study is that we lack a comprehensive understanding of the complex action of NO in the blood and CSF in the acute stage of ischemic stroke. The initial endothelial NO expressed in the blood might 
exhibit protective qualities, which is consistent with its ability to improve microhaemorheology [28]. Additionally, whether the CSF cytokine levels are dependent upon serum/blood concentrations and the blood-CSF barrier function and whether the CSF markers are synthesized purely intrathecally has yet to be evaluated.

\section{Conclusions}

The results of the present investigation demonstrate that nitrate $\left(\mathrm{NO}_{2}\right)$ content in the CSF appears to reflect the severity of the oxidative stress reaction that develops in the ischemic neurovascular unit in the first hours of stroke and can predict functional outcome. CSF IL-6 content seems to be the most reliable prognostic indicator in the acute phase of ischemic stroke, with regard to the probability of infarct size, the clinical course of disease and the functional outcome of stroke at one month.

\section{Acknowledgements \\ Study is supported by an INTAS collaborative research grant and the Georgian Academy of Sciences. The sponsors had no role in study design, data collection, data analysis, data interpretation, or writing of the manuscript.}

\section{Author details \\ 'Department of Neuromedicine, Tbilisi State Medical University, Tbilisi, Georgia. ${ }^{2}$ Department of Biophysics and Biochemistry, Tbilisi State Medical University, Tbilisi, Georgia. ${ }^{3}$ Department of Neurology, Tel-Aviv Sourasky Medical Center, Sackler Faculty of Medicine, Tel-Aviv University, Tel-Aviv, Israel.}

\section{Authors' contributions}

MB designed the study, interpreted the data and drafted the manuscript. TS carried out the immunoassays and EPR analysis. RS participated in acquisition of data, interpretation and statistical analysis. NI conducted the additional statistics and interpreted the data. NB revised critically the intellectual content and gave the final approval for the given version of manuscript.

All authors read and approved the final manuscript.

\section{Authors' Information}

MB Associated Professor of Neurology MD, PhD. Department of Neuromedicine, Tbilisi State Medical University, Tbilisi, Georgia. TS Full Professor of Biophysics PhD. Department of Biophysics and Biochemistry, Tbilisi State Medical University, Tbilisi, Georgia

RS Full Professor of Neurology MD, PhD. Department of Neuromedicine, Tbilisi State Medical University, Tbilisi, Georgia.

$\mathrm{NI}$ Associated Professor of Biophysics, PhD, Head of Medical Statistics Section of the Department of Biophysics and Biochemistry, Tbilisi State Medical University, Tbilisi, Georgia

NB Full Professor of Neurology, MD. Department Head, Tel-Aviv Sourasky Medical Center, Sackler Faculty of Medicine, Tel-Aviv University, Tel-Aviv, Israel

\section{Competing interests}

The authors declare that they have no competing interests.

Received: 1 September 2010 Accepted: 30 March 2011

Published: 30 March 2011

\section{References}

1. Aktas O, Ullrich O, Infante-Duarte C, Nitsch R, Zipp F: Neuronal damage in brain inflammation. Arch Neurol 2007, 64(2):185-189.
2. Cherubini A, Ruggiero C, Polidori MC, Mecocci P: Potential markers of oxidative stress in stroke. Free Rad Biol Med 2005, 39:841-852.

3. The European Stroke Organization (ESO) Executive Committee and the ESO Writing Committee: Guidelines for Management of Ischaemic Stroke and Transient Ischaemic Attack. Cerebrovasc Dis 2008, 25:457-507.

4. Adams HP, Bendixen BH, Kapelle $L$, the TOAST investigators: Classification of subtypes of acute ischemic stroke. Definitions for use of multicenter clinical trial TOAST. Stroke 1993, 24:35-41.

5. Jood K, Redfors $P$, Rosengren A, Blomstrand $\mathrm{CH}_{\text {, Jern }} \mathrm{CH}$ : Self-perceived psychological stress and ischemic stroke: a case-control study. BMC Medicine 2009, 7:53.

6. Truelsen T, Nielsen N, Boysen G, Grønbæk M: Self-Reported Stress and Risk of Stroke, The Copenhagen City Heart Study. Stroke 2003, 34:856-862.

7. Adams HP, et al: NIHSS and Patient Outcomes. Neurology 1999, 53:126-131.

8. Dromerick AW, Edwards DF, Diringer MN: Sensitivity to changes in disability after stroke: A comparison of four Scales useful in clinical trials. Journal of Rehabilitation Research and Development 2003, 40(1):1-8.

9. Govan L, Langhorne P, Weir CJ: Categorizing stroke prognosis using different stroke scales. Stroke 2009, 40(10):3396-9.

10. Fink B, Fink N: EPR techniques for detection of nitric oxide in vivo as an index of endothelial disfunction. Pharmacological reports 2006, 58:8-15.

11. Richardson SRussell, Donato AJ, Uberoi AD, Wray W, Lawrenson L, Nishiyama S, Bailey DM: Exercise-induced brachial artery vasodilation: role of free radicals. Am JPhysiol Heart Circ Physiol 2007, 292:1516-1522.

12. Guevara I, Iwanejko J, Dembinska-Kiec A, et al: Determination of nitrite/ nitrate in human biological material by the sample Griess reaction. Clin Chim Acta 1998, 274:177-88.

13. Schroeter M, Jander S, Witte OW, Stoll G: Local immune responses in the rat cerebral cortex after middle cerebral artery occlusion. $J$ Neuroimmunol 1994, , 55: 195-203.

14. Fassbender K, Rossol S, Kammer T, et al: Proinflammatory cytokines on the serum of patients with acute cerebral ischemia, kinetics and secretion and relation to the extent of brain damage and outcome of disease. $J$ neurol Sci 1994, 122:135-139.

15. Garcia JH, Liu KF, Relton JK: Interleukin-1 receptor antagonist decreases the number of necrotic neurons in rats with middle cerebral artery occlusion. Am J Pathol 1995, 147:1477-1486.

16. Chamorro A, Hallenbeck J: The harms and benefits of inflammatory and immune responses in vascular disease. Stroke 2006, 37:291-293.

17. Smith CJ, Emsley HCA, Gavin CM, et al: Peak plasma interleukin-6 and other peripheral markers of inflammation in the first week of ischemic stroke correlate with brain infarct volume. stroke severity, and long-term outcome. BMC Neurol 2004, 4:2-9.

18. Emsley HCA, Smith CJ, Georgiou RF, Vail A, Tyrrell P-J, Barberen E-M, et al: Correlation of systemic inflammatory response with infarct volume in acute ischemic patients Response. Stroke 2005, 36(2):228-229.

19. Barone FC, Arvin B, White RF, et al: Tumor necrosis factor-a a mediator of focal ischemic brain injury. Stroke 1997, 28:1233-1244.

20. Martin-Villaba A, Herr I, Jeremias I, et al: CD95 ligand (Fas-L/APO-1L) and tumor necrosis factor-related apoptosis-inducing ligand mediate ischemia-induced apoptosis in neurons. J Neurosci 1999, 19:3809-3817.

21. Dietrich ND, Busto R, Bethea JR: Postischemic hypothermia and IL-10 treatment provide long-lasting neuroprotection of CA1 hippocampus following transient global ischemia in rats. Exp Neurol 1999, 158:444-450.

22. Spera PA, Ellison JA, Feurstein GZ, Barone FC: IL-10 reduces rat brain injury following focal stroke. Neurosci Lett 1998, 251:189-192, 24.

23. Chong WL, Sengupta A, Sandercock P: Blood Markers for the Prognosis of Ischemic Stroke: A Systematic Review. Stroke 2009, 40:380-389.

24. Kunz A, Park L, Abe T, Gallo EF, Anrather J, Zhou P, ladecola C: Neurovascular protection by ischemic tolerance: Role of Nitric Oxide and Reactive Oxygen Species. J Neurosci 2007, 27(27):7083-7093.

25. Moncada S, Higgs A: The L-arginine-nitric oxide pathway. N Engl J Med 1993, , 329:: 2002-2012

26. Ignarro $\amalg$ : Signal transduction mechanisms involving nitric oxide. Biochem Pharmacol 1991, 41:485-490.

27. Orio D, Kunz A, Kawano T, Anrather J, Zhou P, ladecola C: Lipopolysaccharide Induces Early Tolerance to Excitotoxicity via Nitric Oxide and cGMP. Stroke 2007, 38(10):2812-2817

28. Lavi SH, Egbarya R, Lavi R, Jacob G: Role of Nitric Oxide in the Regulation of Cerebral Blood Flow in Humans. Circulation 2003, 107:1901. 


\section{Pre-publication history}

The pre-publication history for this paper can be accessed here:

http://www.biomedcentral.com/1471-2377/11/41/prepub

doi:10.1186/1471-2377-11-41

Cite this article as: Beridze et al: Selected acute phase CSF factors in

ischemic stroke: findings and prognostic value. BMC Neurology 2011

11:41.

Submit your next manuscript to BioMed Central and take full advantage of:

- Convenient online submission

- Thorough peer review

- No space constraints or color figure charges

- Immediate publication on acceptance

- Inclusion in PubMed, CAS, Scopus and Google Scholar

- Research which is freely available for redistribution 